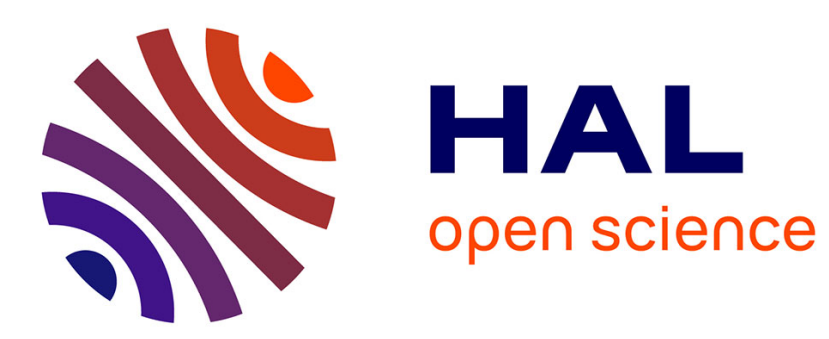

\title{
Torsional shear flow of granular materials: shear localization and minimum energy principle
}

\author{
Riccardo Artoni, Patrick Richard
}

\section{To cite this version:}

Riccardo Artoni, Patrick Richard. Torsional shear flow of granular materials: shear localization and minimum energy principle. Computational Particle Mechanics, 2018, 5 (1), pp. 3-12. 10.1007/s40571016-0143-2 . hal-01679761

\section{HAL Id: hal-01679761 https://hal.science/hal-01679761}

Submitted on 10 Jan 2018

HAL is a multi-disciplinary open access archive for the deposit and dissemination of scientific research documents, whether they are published or not. The documents may come from teaching and research institutions in France or abroad, or from public or private research centers.
L'archive ouverte pluridisciplinaire HAL, est destinée au dépôt et à la diffusion de documents scientifiques de niveau recherche, publiés ou non, émanant des établissements d'enseignement et de recherche français ou étrangers, des laboratoires publics ou privés. 


\title{
Torsional shear flow of granular materials: shear localization and minimum energy principle
}

\author{
Riccardo Artoni · Patrick Richard
}

October 26, 2016

\begin{abstract}
The rheological properties of granular matter submitted to torsional shear are investigated numerically by means of Discrete Element Method. The shear cell is made of a cylinder filled by grains which are sheared by a bumpy bottom and submitted to a vertical pressure which is applied at the top. Regimes differing by their strain localization features are observed. They originate from the competition between dissipation at the sidewalls and dissipation in the bulk of the system. The effects of the (i) the applied pressure (ii) sidewall friction and (iii) angular velocity are investigated. A model, based on the purely local $\mu(I)$-rheology and a minimum energy principle is able to capture the effect of the two former quantities but unable to account the effect of the latter. Although, an ad-hoc modification of the model allows to reproduce all the numerical results, our results point out the need for an alternative rheology.
\end{abstract}

\section{Introduction}

The rheology of granular materials is relevant to many industrials applications (grain transport and storage) and to natural events (avalanches, mud-slides...). Several geometries have been used to probe the rheology of granular systems (inclined plane, shear cell, confined gravity-driven flows...) but, the full description of $3 \mathrm{D}$ flows remains challenging.

In this work we present discrete element simulations of a wall-bounded three dimensional dense granular flow. The flow configuration studied in this paper, which can be referred as torsional shear flow, is a rather

LUNAM Université, IFSTTAR, MAST, GPEM, F-44340 Bouguenais, France

E-mail: riccardo.artoni@ifsttar.fr classical one in rheometry, which was rarely used for studying granular materials. It consists in a cylindrical geometry filled by the studied system, where the bottom wall rotates and the upper and cylindrical wall are fixed. In this work the flow is at fixed normal stress, i.e. the upper wall is free to move vertically under the action of the imposed normal force and the reaction of the particles contained in the cylinder. This configuration is interesting because simple velocity profiles were obtained for viscous and viscoelastic fluids, and therefore it is tempting to consider it as a granular rheometer. On the other hand, secondary flows were already observed for newtonian fluids in this geometry, and it is therefore interesting to see if they can develop also for granular flows. As it will be clear in the following, this configuration displays shear localization. Shear localization is a fundamental property of granular systems. Examples of configurations displaying such a phenomenon are the vertical chute flow [1-3], the cylindrical Couette cell [4], and the different types of split-bottom shear cells [5-9]. Such configurations are an important benchmark for granular flow modeling, and are therefore precious for evaluating the performance of the different rheologies proposed in the Literature. A large body of work in the last decade has been devoted to the so-called $\mu(I)$ rheology $[4,10]$. While this rheology seems to work well (and should be probably better referred to) as an empirical, macroscopic scaling law, its colinear extension to 3D [11] was shown to have some drawbacks for complex flows, particularly when approaching the quasistatic regime of flow $[12,13,6]$. These problems seem to be related to the local nature of the $\mu(I)$ rheology and motivated research on nonlocal models of granular flows such as fluidity-based models [14-17] and models inspired by kinetic theories [18-20]. The main objective of this work is therefore to characterize the granu- 
lar flow in such a configuration and to discuss a simple modeling. So, a particular focus will be given on the kinematics of the flow and on the flow regimes as a function of the main system parameters.

The outline of this paper is the following. We begin this article with a brief description of the flow configuration and of the numerical methods used (section 2). We then report in section 3 the velocity profiles obtained under several conditions. Section 4 is devoted to the exploitation of the data and investigate how they can be modeled. Finally we present our conclusions.

\section{Flow configuration and simulation method}

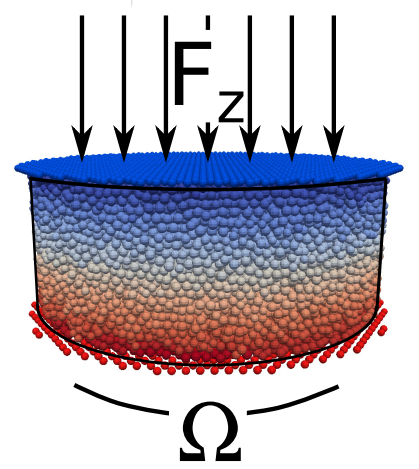

Fig. 1 Sketch of the torsional flow configuration. Grains fill a cylindrical vessel with smooth but frictional sidewalls. The bottom and upper walls are made bumpy by gluing grains on their surfaces. A vertical force $F_{z}$ is applied to the latter wall which is free to move vertically according to the applied forces. On the contrary, the former wall only rotates at an angular velocity $\Omega$.

Numerical simulations are performed using the non smooth contact dynamics method [21], as implemented in the LMGC90 open source framework [22].

As already introduced, the torsional shear flow configuration (sketched in Fig. 1) is a cylinder (radius $R=$ $12 d$, variable height $H$ ) filled with a granular material, where the bottom boundary is rotating, while a normal force is applied by the top wall. The top and bottom walls are bumpy, while the cylindrical wall is smooth but frictional. Gravity acts on the system along $z$. The top wall cannot move on the $x$ - and $y$ - directions but is free to move in the $z$-direction, simply according to the balance between its weight, the externally applied force and the force exerted by the grains. Simulations were performed with $N=10000$ slightly polydisperse spheres (uniform number distribution in the range $0.9 d-1.1 d$ ) interacting through perfectly inelastic collisions and Coulomb friction $\left(\mu_{p}=0.5\right)$. Each bumpy wall was composed of 100 spheres with the same properties of the particles. The coefficient of restitution has nearly no influence on dense granular flows due to the presence of enduring contacts [23]. Consequently, we chose perfectly inelastic grains to maximize dissipation and thus save computation time. Interactions of particles with the flat walls were also perfectly inelastic and frictional (with a coefficient of friction $\mu_{p w}$ ).

We performed several simulations varying the angular velocity of the bottom bumpy wall $\Omega$, the force applied to the upper bumpy wall $F_{z}$, and the particle wall friction coefficient $\mu_{p w}$. The first two parameters can be made dimensionless for example by considering a particle Froude number $\tilde{\Omega}=\Omega R / \sqrt{g d}$ and the ratio between the total force mass exterted by the top wall and the weight of the grains, $\tilde{F}=\frac{M g+F_{z}}{N m g}$ where $m$ is the average particle mass, and $M=100 \mathrm{~m}$ is the mass of the top wall. In particular, the investigated ranges correspond to $\mu_{p w}=0-0.3, \tilde{\Omega}=0.12-2.4, \tilde{F}=0.2-100$. We did not perform every possible permutation of the three variables but we chose some representative sets of the couple $(\tilde{\Omega}, \tilde{F})$ and varied the wall friction coefficient for each set. Table 1 resumes the sets chosen. In order to understand to which flow regime our simulations belong to, it is useful to introduce a dimensionless number, the inertial number, defined as $I=\dot{\gamma} d / \sqrt{p / \rho}[4,10]$, where $\dot{\gamma}$ is the shear rate and $p$ is a pressure, while $\rho$ is particle density. However in our simulations the shear rate is an increasing function of the radial coordinate, so will be the inertial number. Shear localization and the presence of stress profiles will also induce $z$ variation of $I$. In order to compare simulation sets we therefore define a characteristic inertial number $I_{c}$ by considering the average shear rate at $r=R, \Omega R / H$, and the theoretical pressure at mid-height, $(\tilde{F}+1 / 2) N m g /\left(\pi R^{2}\right)$. $I_{c}$ may give a coarse estimate of the expected maximum value of $I$ for each set. From Table 1 we can conclude that our simulations lie in a range of inertial number from 0 (for $r=0$ ) to $10^{-1}$, which corresponds to the quasistatic and dense regimes of flow. Set 6 in Table 1 was explicitely chosen because, if the behavior of the system was described by this characteristic inertial number, decreasing the rotational velocity by one order of magnitude should be the same as increasing the pressure by two orders of magnitude (i.e. set 1 would behave like set 6$)$.

In the contact dynamics method the choice of the time step is not related to the elasticity of the particles but to the other characteristic times of the system. Three characteristic times may be defined: the characteristic time related to gravity, $\sqrt{d / g}$, the characteristic time related to particle velocities, $d / V$, and the characteristic time related to pressure, $\frac{d}{\sqrt{p / \rho_{p}}}$ where $\rho_{p}=\frac{6 m}{\pi d^{3}}$ 


\begin{tabular}{|c|c|c|c|}
\hline Set & $\tilde{\Omega}$ & $\tilde{F}$ & $I_{c}$ \\
\hline 1 & 0.12 & 0.2 & $210^{-3}$ \\
2 & 0.24 & 0.2 & $410^{-3}$ \\
3 & 1.2 & 0.2 & $210^{-2}$ \\
4 & 2.4 & 0.2 & $410^{-2}$ \\
5 & 1.2 & 8 & $610^{-3}$ \\
6 & 1.2 & 100 & $210^{-3}$ \\
\hline
\end{tabular}

Table 1 Sets of $\tilde{\Omega}$ and $\tilde{F}$ used in the simulations. For each set the wall friction coefficient was varied in the range $\mu_{p w}=0-0.3$. The last column displays an estimate of the global inertial number for each set.

is the particle density. The time step must be choosen smaller than such characteristic times. For estimating the extremal values of the characteristic times we considered the maximum expected velocity of the particles $(\Omega R)$ and the pressure at the bottom wall $\left(\frac{(\tilde{F}+1) N m g}{\pi R^{2}}\right)$. Given that our simulations were performed in the dense regime of flow, the characteristic time related to pressure is the controlling one and spans in the range $210^{-2}$ $210^{-1}$. The time step was therefore chosen as $\Delta t=$ $210^{-4}$ for all the simulations. Each simulation was performed in parallel (OpenMP) on 4 cores (2.7Ghz) for approximately 30 days. The time needed for reaching a stationary state (determined by the kinetic energy evolution) was nearly independent on system parameters, and we found $T_{s s} \approx 10 \sqrt{d / g}$. The convergence rate was lower when increasing pressure and when decreasing the velocity, given that the relative number of long lasting contacts was higher in those cases.

Averaging method. An averaging technique is needed to obtain estimates of continuum-like variables such as velocity, solid fraction, stresses from their discrete counterparts. Several frameworks have been proposed in the literature [24-28]; recent works have highlighted the effect of the coarse-graining length [29] and the subtleties behind the calculation of kinetic terms [28]. In this work, we report results on azimuthal velocity profiles. In order to compute such profiles, several snapshots of particle positions and velocities were extracted from the simulations at different times. Due to the axial symmetry of the flow geometry, averages were computed with respect to a grid in the $(r, z)$-plane; for each grid point the average velocity was computed by the space-time weighted average $[25,28]$ :

$\mathbf{v}(\mathbf{x}, t)=\frac{\int_{-\infty}^{\infty} \sum_{p} w_{p} m_{p} \mathbf{v}_{p} d t^{\prime}}{\int_{-\infty}^{\infty} \sum_{p} w_{p} m_{p} d t^{\prime}}$

where $m_{p}$ and $\mathbf{v}_{p}$ are respectively the mass and velocity of particle $p$, and $w_{p}=w\left(\mathbf{x}_{p} \mathbf{x}, t^{\prime}-t\right)$ is a weighting function. As for the latter, due to the existence of a stationary state, the time average was performed as a simple average of the space-weighted averages; for space averaging, on the other hand, a Heaviside step function around the grid point with diameter equal to one particle diameter was chosen. This corresponds to performing averages on toroidal volumes. A similar averaging procedure was already discussed by Luding [30]. When dealing with such a complex averaging domain, it is important to remember that the mass is not evenly distributed in the radial dimension: therefore the average velocity has to be referred not to center of the torus but to its gyration radius.

\section{Results}

In the following we will focus on the kinematics displayed by the torsional shear cell, and in particular we attempt a characterization of the shear localization features of this flow configuration. A full characterisation of the system in terms of the other variables (stresses, fluctuating energy balance, wall fields) will be given elsewhere. It has to be noted that an analysis of wall friction and wall slip in a similar geometry has recently appeared [31].

Autosimilarity of the profiles. Figure 2 displays velocity profiles along $z$ for different values of the distance from the axis of symmetry $r$, for the reference case $\mu_{p w}=0.3$, $\tilde{\Omega}=1.2, \tilde{F}=0.2$. As expected, due to the torsional motion, the azimuthal velocity of the grains depends on $r$. However, when rescaled by the azimuthal velocity of the bottom wall for the same $r$, which is $\Omega r$, velocity profiles nearly collapse on the same curve, which corresponds to a localization near the bottom wall, and an exponential decay. From the bottom inset of Figure 2 we can see that the velocity profile, when rescaled by $\Omega r$, indeed displays a small dependence on $r$, due to the fact that the cylindrical wall slightly slows down the particles. In particular, the shear band width slightly decreases with $r$ near the cylindrical wall. This effect is however quite small and decreases when decreasing wall friction (not shown). We can therefore conclude that velocity profiles are nearly autosimilar with respect to $r$ and can be written as $v_{\theta}(z)=\Omega r f(z)$, where $f(z)$ is a similarity function.

Effect of wall friction Figure 3 shows the effect of the wall friction coefficient (between flowing particles and the cylindrical wall) on the flow profiles, for $\tilde{\Omega}=1.2$, $\tilde{F}=0.2$. For the sake of simplicity, due to the nearly autosimilarity of the profiles, the profiles displayed in the Figure correspond to the average of the rescaled velocity profiles, $v_{\theta} / \Omega r$. As said above, profiles display shear localization with an exponential decay. However, the localization pattern depends strongly on wall friction. For 


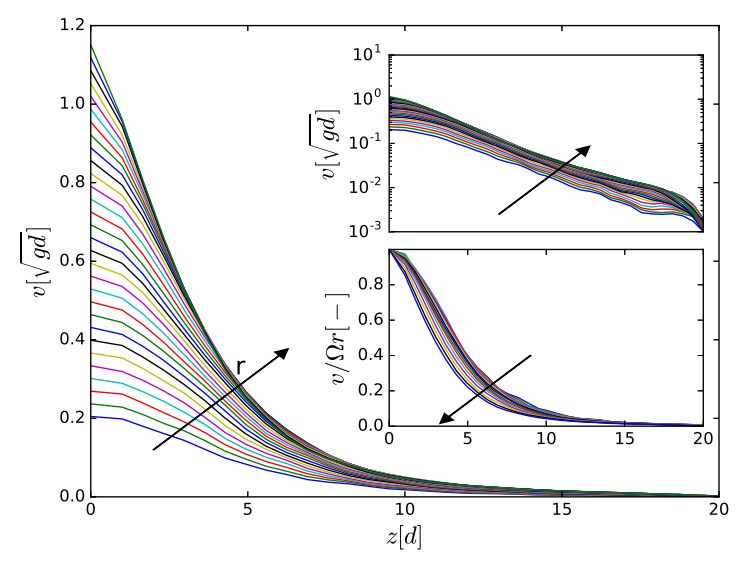

Fig. 2 Azimuthal velocity profiles for different $r$ values, for the reference case $\mu_{p w}=0.3, \tilde{\Omega}=1.2, \tilde{F}=0.2$. Insets: (top) lin-log plot displaying exponential profiles, (bottom) normalized velocity profiles. Velocity profiles are exponential and autosimilar.

zero to low values of the wall friction coefficient, shear is localized near the top wall, while for strong friction shear is localized near the bottom wall. For intermediate values of the wall friction coefficient, a more uniform velocity profile prevails. It can be shown that the velocity profile is a combination of exponentials, of the form

$v_{\theta}=\Omega r \frac{\alpha^{z / d-H / d}-\beta^{H / d-z / d}}{\alpha^{z_{0} / d-H / d}-\beta^{H / d-z_{0} / d}}$,

where $\alpha$ and $\beta$ are dimensionless parameters. Note that $0<\alpha, \beta<1 ; z_{0}$ is needed for fitting because the velocity profile may equal $\Omega r$ sligthly above the bottom wall position. $z_{0}$ is of order of half a particle diameter. The change in the localization pattern with wall friction was already discussed in a similar geometry [31], and remains an interesting aspect which needs to be explained.

Effect of confining pressure Figure 4 shows the effect of the normal force applied to the top wall on the flow profiles, for $\tilde{\Omega}=1.2$, and three different values of the wall friction coefficient. As the wall friction coefficient, confinement pressure has a strong effect on velocity profiles: for negligible wall friction, increasing the normal force widens the top localized shear band. We can imagine that for very large confinement pressures and no wall friction the velocity profiles will become linear. On the other hand, for larger values of the wall friction coefficient, increasing the normal force induces a strengthening of the bottom localization pattern with a decrease in the shear band width. Similarly, Singh et al [6] reported a decrease in the shear band width when increasing gravity in a split bottom cell.

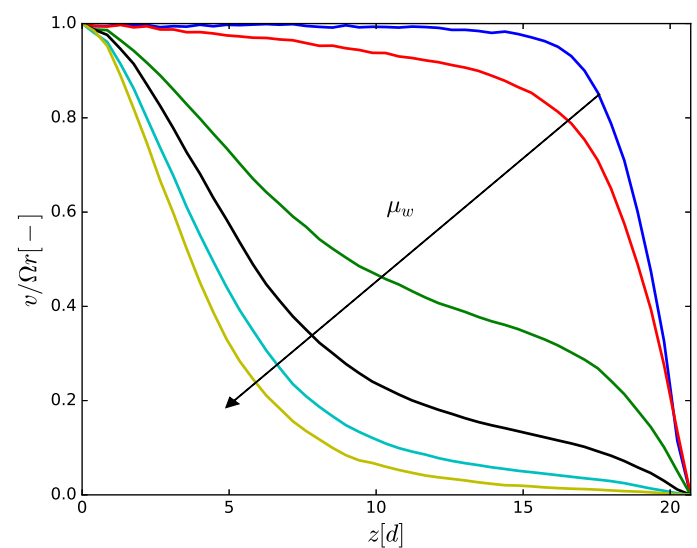

Fig. 3 Average normalized azimuthal velocity profiles as a function of wall friction, for $\tilde{\Omega}=1.2, \tilde{F}=0.2$, and different values of the wall friction coefficient: $\mu_{p w}=0,0.1,0.13,0.15,0.2,0.3$.

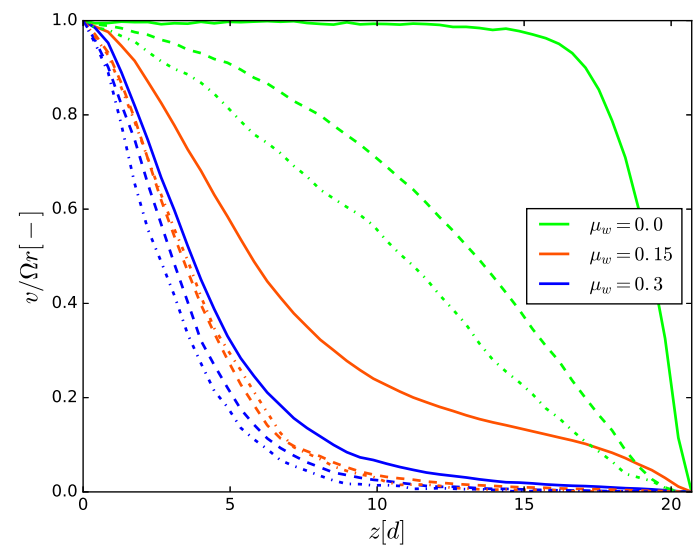

Fig. 4 Average normalized azimuthal velocity profiles as a function of applied pressure, for $\tilde{\Omega}=1.2$, and three different values of the wall friction coefficient, and three different values of the force applied to the top wall : $\tilde{F}=0.2$ (solid lines), $\tilde{F}=8$ (dashed lines), $\tilde{F}=100$ (dot-dashed lines).

Effect of angular velocity Figure 4 shows the effect of the rotating velocity of the bottom wall on the flow profiles, for $\tilde{F}=0.2$, and three different values of the wall friction coefficient. Surprisingly, in the range of velocities considered here, the driving speed has little effect on the shape of velocity profiles. This is a feature common to other granular flows, such as for example the vertical chute flow $[2,3,1]$, where the shape of the velocity profile does not depend on flowrate. Koval et al [32] reported numerical simulations for a $2 \mathrm{~d}$ annular Couette cell displaying constant shear band width in the quasistatic limit and increasing shear band width when approaching the inertial regime. Given the nature of our configuration and the range of I studied, nearly all the flow is in the quasistatic regime, so this is probably the reason for the independence of the velocity profiles on 
the angular velocity. On the other hand, Jop [9] solved the $\mu(I)$ rheology for the split bottom cell with free surface and found that the theory predicted an increasing shear band width. This has not been tested against data to our knowledge, so it is difficult to judge about a possible contradiction, especially considering that our geometry is different in many aspects from Jop's one. There's definitely a strong need for investigation on the effect of velocity on shear bands in many flow configurations, in view of the fact that shear bands are an important benchmark for granular flow theories.

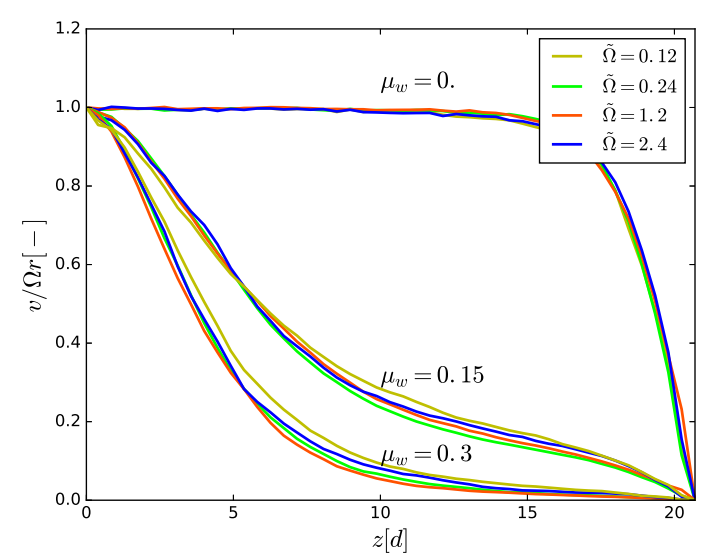

Fig. 5 Average normalized azimuthal velocity profiles as a function of angular velocity of the bottom wall, for $\tilde{F}=0.2$, and three different values of the wall friction coefficient.

It is useful at this point to resume the main results obtained from the simulations. For the range of parameters considered, we have found:

- nearly autosimilar and rate independent azimuthal velocity profiles: $v_{\theta} / \Omega r=f(z)$ seems to depend only slightly on $r$ and $\Omega$.

- for low friction and low pressure, velocity profiles display a plug flow with shear localization near the top wall

- for high friction, velocity profiles display shear localization near the bottom wall, with the development of a creep zone far from the shear band.

- for negligible friction and high pressure we seem to tend to a fully sheared situation.

A tentative sketch of the regimes of our system as a function of $\tilde{F}$ and $\mu_{p w}$ is given in Figure 6. A more complete parametrical study has to be performed in order to explore the limits of such a regime map, and to characterize the transition zones in detail. In the following we will try to see if a modeling framework based on simple rheological considerations and a least effort principle can predict the behaviors listed above.

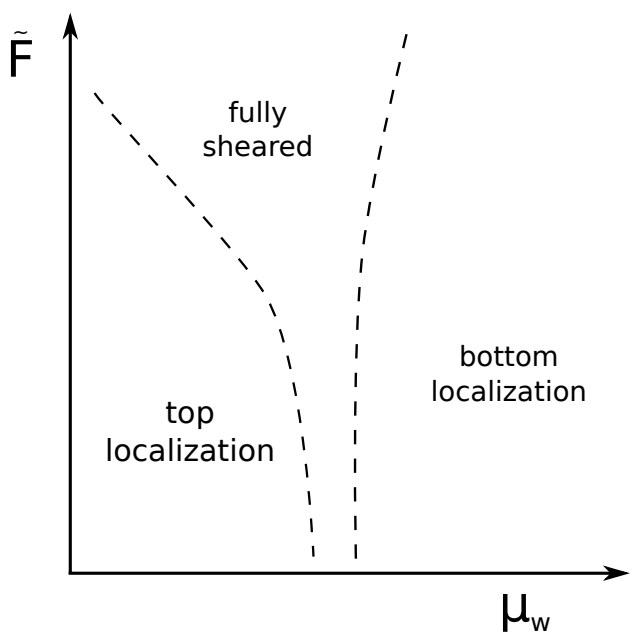

Fig. 6 Hypothetical flow regime map as can be drawn from present results.

\section{Modeling}

In the previous section we discussed numerical results concerning the torsional shear flow of granular materials, with a particular focus on the effect of wall friction, confining pressure and driving velocity. Results are interesting and provide a valuable benchmark for granular flow models. A simple approach would be to solve the momentum balance equations with the $\mu(I)$ rheology $[33,9]$. Here we prefer here to adopt a more robust model, employing some of the information coming from the $\mu(I)$ rheology, but in the form of a minimum energy principle. This is also motivated by the fact that, as shown recently in a similar geometry [31], boundary conditions for the velocity field and wall stresses can be tricky for the smooth wall. It should be pointed out here that our aim is to derive a model capable to reproduce qualitatively our numerical results and thus identify the physical mechanisms which explain the different observed regimes.

The rate of work transferred to the system by the rotating bottom wall can be divided in two contributions: (1) the power needed to shear the material in the bulk, and (2) the power needed to let the material slip at the cylinder wall. The first term, which corresponds to the so-called "stress power" in fluid mechanics, is given, per unit volume, by $P_{b u l k}=\sigma_{i j} e_{i j}$, which is the contracted product of the stress tensor $\sigma_{i j}$ and the rate of deformation tensor $e_{i j}=0.5\left(v_{i, j}+v_{j, i}\right)$. We briefly recall that using the classical decomposition $\sigma_{i j}=-p \delta_{i j}+\tau_{i j}$ where $\delta_{i j}$ is the Kronecker delta, $p=1 / 3 \sigma_{i i}$ the pressure, $\tau_{i j}$ the deviatoric stress tensor, the stress power becomes $P_{b u l k}=\tau_{i j} e_{i j}-p e_{k k}$, and that the second term disappears for isochoric motion. If, for the purpose of deriving a minimum energy principle, we assume co- 
linearity of the stress-rate of strain relationship, the stress power is simply given by $P_{b u l k}=|\tau||\dot{\gamma}|$, where $|\tau|=\sqrt{0.5\left(\tau_{i j} \tau_{i j}\right)}$ is the norm of the deviatoric stress tensor, and $|\dot{\gamma}|=\sqrt{2\left(e_{i j} e_{i j}\right)}$ is the shear rate as defined by Bird [34]. Let's assume a simple scaling for stresses in granular flow, i.e. the $\mu(I)$ scaling $[4,10]$. This scaling law states that the shear stress is proportional to the pressure via a dimensionless quantity $\mu$, which is a function of the already discussed inertial number $I$ :

$$
\frac{\|\tau\|}{p}=\mu(I)=\mu_{0}+b I,
$$

where $\mu_{0}$ and $b$ are material constants. Introducing such an expression, we obtain that the local, bulk stress power per unit volume is $P_{b u l k}=\mu(I) p|\dot{\gamma}|$. Note that this scaling is purely local: it correlates the stress at a given position to the shear rate at the same position. Consequently, it cannot capture the cooperative effects that prevail to describe the behavior of granular materials in quasi-static flowing zones for which the bulk stress/strain-rate behavior appears to vary with the macroscopic geometry. As regards the second contribution to the total power, it is straightforward to see that the rate of work required to maintain steady slip at the cylindrical wall per unit surface is given by $P_{w a l l}=\mu_{w} \sigma_{r r} v_{s l i p}$, where $\mu_{w}$ is a wall friction coefficient and $v_{\text {slip }}$ is the local slip velocity. If, for the sake of simplicity, we hypothesize zero normal stress difference, all the three normal stresses are equal, i.e. $\sigma_{r r}=\sigma_{\theta \theta}=\sigma_{z z}=p$, this term becomes $P_{\text {wall }}=\mu_{w} p v_{\text {slip }}$. Under the assumptions detailed above, the total power transferred to the system is therefore given by the sum of the two terms respectively integrated on the volume and the wall surface, that is

$$
\begin{aligned}
\mathcal{P}_{\text {tot }} & =\mathcal{P}_{\text {bulk }}+\mathcal{P}_{\text {wall }} \\
& =\int \mu(I) p|\dot{\gamma}| d V+\int \mu_{w} p v_{\text {slip }} d S .
\end{aligned}
$$

We will close our model by making the assumption that the system selects the velocity profile which requires the least total power [35, 7]. The relevance of a least effort principle can be measured by the following reasoning. Due to the presence of gravity, stresses are expected to increase with depth, and therefore $\mathcal{P}_{\text {bulk }}$ is higher when shear is localized at the bottom. On the other hand, $\mathcal{P}_{\text {wall }}$ is higher if shear is localized near the top wall, because the plug flow zone contributes largely to the power needed to let particles slide at the wall, given that nearly all the particles at the wall slip with a large velocity. Changing the system parameters may change the relative balance of the two terms and therefore the shear localization pattern.
Let's try to develop the terms in Eq. 4. Numerical data showed that velocity profiles were nearly autosimilar. It is easy to show that, if $v_{\theta}(r)$ is linear, the rate of deformation tensor has only one non negligible component, $e_{\theta z}=e_{z \theta}$. The shear rate therefore reduces to $|\dot{\gamma}|=\partial_{z} v_{\theta}=\Omega r\left|f^{\prime}(z)\right|$, where $f(z)=v_{\theta} /(\Omega r)$ is the similarity function defined in Section 3. We assume that the pressure profile is a hydrostatic one: $p(z)=$ $p_{H}-\rho g(z-H)$, where $p_{H}=\left(M g+F_{y}\right) / S=\tilde{F} N m g / S$ is the pressure applied by the top wall.

Considering that $\tilde{F}=\frac{p_{H} S}{N m g}$, and defining the dimensionless total power as $\mathcal{P}^{\prime}=\mathcal{P}_{\text {tot }} /\left(\pi R^{3} H \rho g \Omega\right)$, it can be shown that the latter is given by:

$\mathcal{P}^{\prime}=2 \mu_{w} \frac{H}{R} \Phi_{1}+\frac{2}{3} \mu_{0} \Phi_{2}+\frac{1}{2} b \tilde{\Omega}\left(\frac{d}{H}\right)^{3 / 2} \Phi_{3}$,

where $\Phi_{1}, \Phi_{2}$ and $\Phi_{3}$ are three dimensionless functions which are given by:

$$
\begin{aligned}
& \Phi_{1}=\frac{1}{H} \int_{0}^{H}\left(\tilde{F}+1-\frac{z}{H}\right) f(z) d z, \\
& \Phi_{2}=\int_{0}^{H}\left(\tilde{F}+1-\frac{z}{H}\right)\left|f^{\prime}(z)\right| d z, \\
& \Phi_{3}=H \int_{0}^{H} \sqrt{\tilde{F}+1-\frac{z}{H}}\left|f^{\prime}(z)\right|^{2} d z .
\end{aligned}
$$

In the expression given above, one can respectively identify three terms: the wall friction, the static (yield stress), and the dynamic contribution. It is easy to see that the dimensionless power depends on 3 dimensionless material parameters, $\mu_{0}, \mu_{w}$ and $b$, on 3 systemdependent dimensionless numbers: $\tilde{F}, H / R, \tilde{\Omega}(d / H)^{3 / 2}$, and on the shape of the velocity profile given by the dimensionless similarity function. For the fitting proposed above for $f(z)$, this means that $\mathcal{P}^{\prime}$ depends on $\alpha$ and $\beta$. The least effort principle corresponds then to the assumption that the system selects the shear band width and the localization pattern (i.e. the values of $\alpha$ and $\beta$ ) that require the least effort. Given its nature of correcting parameter, the parameter $z_{0}$ can be neglected in this optimization procedure. In practice, having assumed a value for the parameters of the $\mu(I)$ scaling, we numerically computed the integrals $\Phi_{1}, \Phi_{2}$ and $\Phi_{3}$ and determined the values of $\alpha$ and $\beta$ which minimized the power. In the following we assume $\mu_{0}=0.42$ and $b=2$. which are compatible with the literature about the $\mu(I)$ scaling.

Effect of wall friction. Figure 7 displays profiles of the normalized azimuthal velocity profile (i.e. the similarity function $f(z)$ ) obtained with the optimization procedure for $\tilde{\Omega}=1.2, \tilde{F}=0.2$, and the same values of the 
wall friction coefficient as in Figure 3. It is clear that the model is effective in predicting the effect of wall friction on the velocity profiles, with a prevalence of top localization for low friction and of bottom localization for high friction, passing through a more uniform velocity profile for intermediate friction.

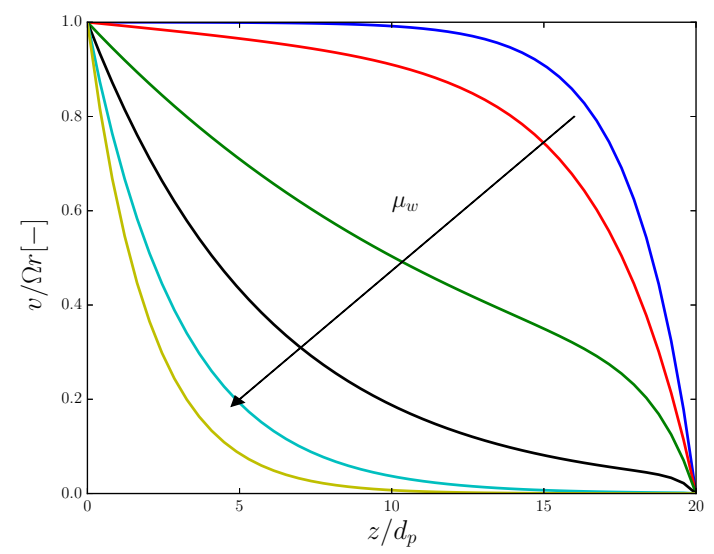

Fig. 7 Average normalized azimuthal velocity profiles as predicted by the minimum energy technique as a function of wall friction, for $\tilde{\Omega}=1.2, \tilde{F}=0.2$, and different values of the wall friction coefficient: $\mu_{w}=0,0.1,0.13,0.15,0.2,0.3$.

Effect of confining pressure. Figure 8, when compared to Fig. 4 shows how also the effect of pressure is well captured by the model: increasing pressure has the effect of decreasing the shear band width for sufficiently high friction, while it increases the shear band width for negligible friction, tending towards a fully sheared situation. Such a tendency is justified by the fact that for low friction $\mathcal{P}_{\text {wall }}$ is negligible and only $\mathcal{P}_{\text {bulk }}$ determines the shear localization pattern. If the confining pressure is very large, there is nearly no pressure profile along $z$, and therefore no reason for shear localization.Therefore the basic ideas behind the $\mu(I)$ scaling, coupled with a minimum energy principle, are able to account for the dependence of shear localization on wall friction and confining pressure.

Effect of rotating velocity. It is tempting to see if the method predicts also the slight effect produced by the driving speed on the velocity profiles seen in Fig. 5. It is clear from Figure 9 that the model predicts a too strong effect of the driving speed with respect to numerical simulations.

One could conclude that the dynamic term in Eq. 5 could be ruled out, but in fact this term is needed in

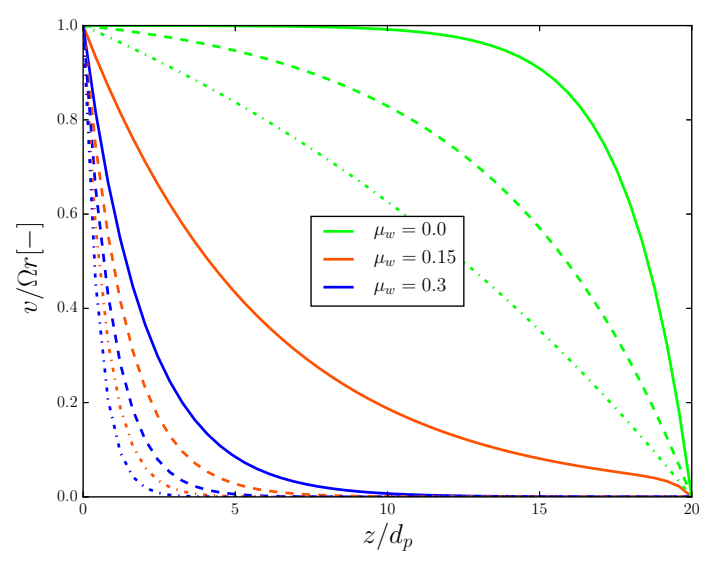

Fig. 8 Average normalized azimuthal velocity profiles as predicted by the minimum energy technique as a function of confining pressure, for $\tilde{\Omega}=1.2$, three different values of the wall friction coefficient, and three different values of the force applied to the top wall : $\tilde{F}=0.2$ (solid lines), $\tilde{F}=8$ (dashed lines), $\tilde{F}=100$ (dot-dashed lines).

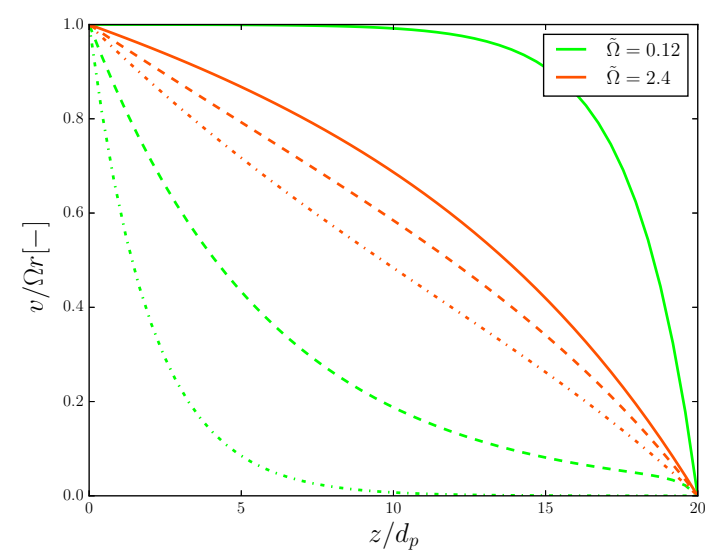

Fig. 9 Average normalized azimuthal velocity profiles as predicted by the minimum energy technique as a function of angular velocity of the bottom wall, for $\tilde{F}=0.2$, and three different values of the wall friction coefficient: $\mu_{w}=0$ (solid lines), $\mu_{w}=0.15$ (dashed lines), $\mu_{w}=0.3$ (dot-dashed lines). Only the two extreme value of the velocity used in the simulations are shown for the sake of clarity.

order to predict a finite shear band width. An ad-hoc expression is therefore proposed here:

$\mathcal{P}^{\prime}=2 \mu_{w} \frac{H}{R} \Phi_{1}+\frac{2}{3} \mu_{0} \Phi_{2}+\frac{1}{2} b \tilde{\Omega}^{m}\left(\frac{d}{H}\right)^{3 / 2} \Phi_{3}$,

in which the velocity dependency is reduced through an exponent $m<1$. The result obtained from the optimization, with $\mu_{0}=0.42, b=2$. and $m=0.05$ is shown in Figure 10. For this parameter set the effect of the other system parameters is recovered too. The optimization leads to a value of the parameter $m$ close to zero because experimental data do not display an effect 
of the rotating velocity. However, the value cannot be zero, otherwise the shear bands would vanish.

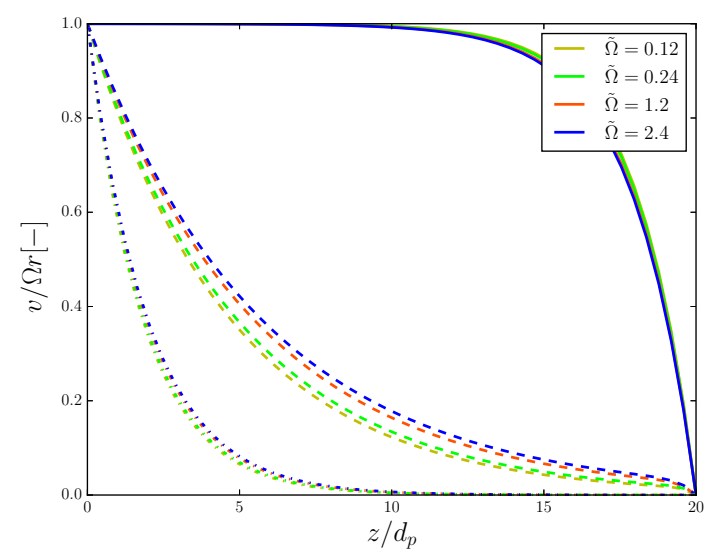

Fig. 10 Average normalized azimuthal velocity profiles as predicted by the modified minimum energy technique as a function of angular velocity of the bottom wall, for $\tilde{F}=0.2$, and three different values of the wall friction coefficient: $\mu_{w}=0$ (solid lines), $\mu_{w}=0.15$ (dashed lines), $\mu_{w}=0.3$ (dot-dashed lines).

Unger et al [35] used a variational approach to model the shear band formation in the split bottom cell. Their model predicted shear bands of vanishing size. This is probably related to the absence of the dynamic contribution in their model. Due to the presence of the dynamic term our model predicts a finite shear band width. However an ad hoc correction is needed to limit the effect of $\Omega$ on the results. The variational approach coupled with the $\mu(I)$ scaling law is thus unable to capture the non-influence of the angular velocity of the bottom wall on the velocity profiles. This can be supported a posteriori by thinking at the combined effect of the rotating velocity and pressure. If the behavior of the system was described only by the characteristic inertial number and the wall friction coefficient, as a simple dimensional analysis could suggest, then we would expect that decreasing the rotational velocity by one order of magnitude should be the same as increasing the pressure by two orders of magnitude. But it is evident that the rotating velocity has no effect, while results are very sensitive to pressure, particularly for low wall friction. This means that the global inertial number and the wall friction coefficient are not sufficient for predicting the behavior of the system. It would be therefore of interest to see if recent modelings of non-locality in granular flows [14-20] are able to capture the full behavior of our system. This will be the subject of a future paper.

Regime maps. With the modified formula for the stress power, Eq. 9, we then look at the combined effect of some parameters. Figure 11 displays the combined effect of pressure and wall friction on the function:

$$
\frac{\Delta}{H}=1-\frac{2}{H}\left|\int_{0}^{H}(f(z)-1+z / H) d z\right|,
$$

which measures the distance from a linear velocity profile, and can provide an estimate of the shear band rescaled by the cell height $H$ if the flow is localized at the top or at the bottom. This regime map displays the already discussed behavior: for low values of both wall friction and applied pressure, shear is localized at the top, while when increasing pressure the range of wall friction implying a bottom localization widens. From the regime map we can speculate that, for very high wall friction and pressure, the model will probably predict a vanishing shear band. This is unlikely, since in granular flows shear bands usually span several particle diameters. In fact the model lacks information on clustering phenomena, which set a lower bound for the shear band width.

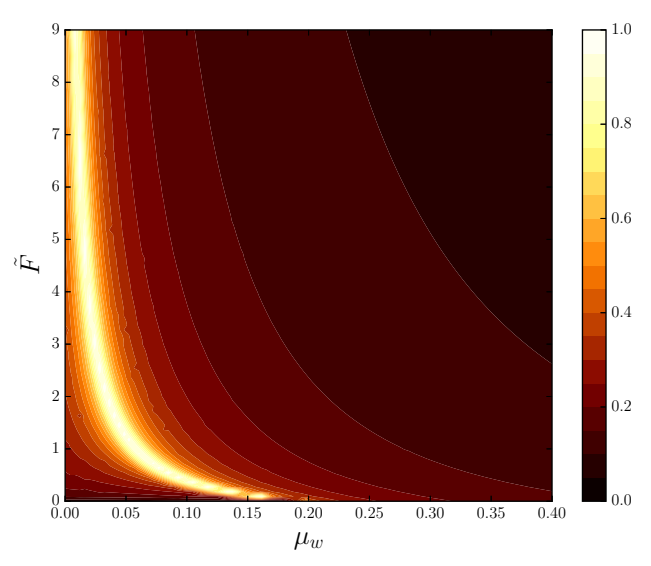

Fig. 11 Map of shear band width (rescaled by the cell height $H)$ as a function of dimensionless pressure $\tilde{F}$ and wall friction coefficient $\mu_{w}$, for $\tilde{\Omega}=1.2$. The bright zone corresponds to a completely sheared material. The region at the left of the bright zone corresponds to top localization, while the one at the right corresponds to bottom localization.

Figure 12 shows the combined effect of pressure and driving speed for two different values of wall friction. It is clear that (thanks to the presence of the exponent $m$ in Eq. 9) the driving speed has a slight effect on the shear band width particularly for large pressures. The present numerical results do not allow to say whether this effect is real or not. Both experiments and simulations for a wider range of rotating velocities of the bottom wall should be performed in order to better understand the effect of the driving speed. 

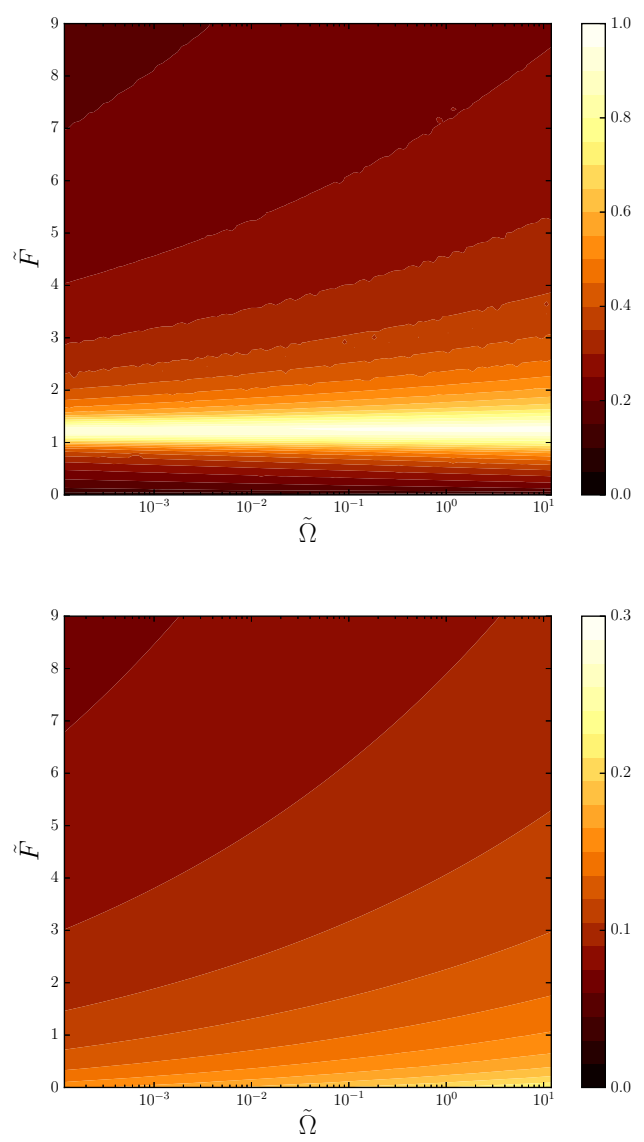

Fig. 12 Maps of shear band width (rescaled by the celll height $H)$ as a function of dimensionless pressure $\tilde{F}$ and dimensionless rotating velocity $\tilde{\Omega}$, for (top) $\mu_{w}=0.05$, (bottom) $\mu_{w}=0.3$. For the top figure, the bright zone corresponds to a completely sheared material. The region below the bright zone corresponds to top localization, while the one above corresponds to bottom localization. For the bottom figure, only bottom localization is present.

Though effective in replicating the numerical simulation results, the optimization technique is based on an expression for the total stress power which was modified ad hoc in order to reduce the velocity dependence of the profiles. A more sound theory is needed in order to better take into account the effect of driving speed and the formation of clusters; however the present approach may be helpful for designing new zones of the parameter space to be investigated and for conceiving experiments on the same flow configuration. An example of this use is Figure 13, in which we plot the combined effect of the depth $H$ and of the wall friction coefficient on the shear band width, for $\tilde{F}=0.2$ and $\tilde{\Omega}=1.2$. It is clear that the model predicts a strong effect of cell height, with top localization for low $H$ and low friction, bottom localization for large $H$ and strong friction, ad a more uniformly sheared profile for intermediate values of the product $H \mu_{w}$. This chart will help us in designing the new simulations and the experiments to be performed in order to study the effect of the cell height.

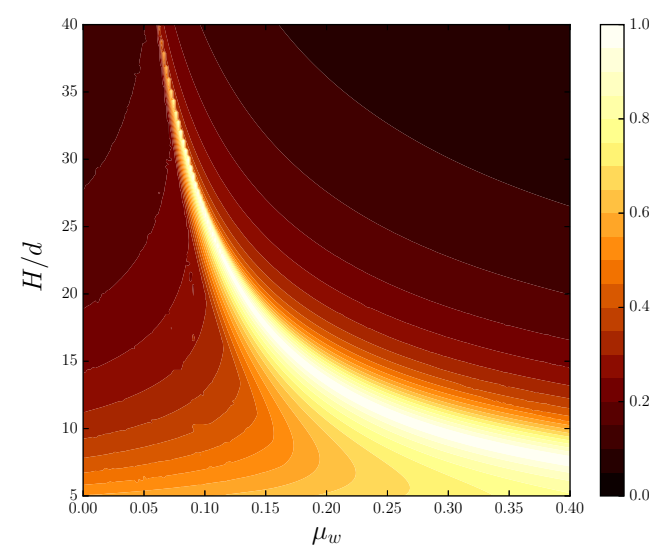

Fig. 13 Map of shear band width (rescaled by the cell height $H$ ) as a function of $H / d$ and wall friction coefficient $\mu_{w}$, for $\tilde{\Omega}=1.2$ and $R / d=12$. The bright zone corresponds to a completely sheared material. The region at the left of the bright zone corresponds to top localization, while the one at the right corresponds to bottom localization.

\section{Conclusions}

Numerical simulations of the torsional shear flow of granular materials was shown to display shear localization. The existence of shear localization, described by an exponential velocity profile, was already reported in similar geometries. For example Orlando \& Shen [36] studied a rapid granular flow in an annular shear cell in which the bottom plate is rotating. They found a top localized shear flow for $H \sim 8-12 d$, and a more uniform shear profile for $H \sim 4-6 d$; by extrapolating their data on shear stress and stress ratio we guess that $\tilde{F}<1$. That is a low pressure which, as shown above in our simulations, coupled with a low wall friction coefficient, could favour a top localization pattern. The original feature of our results is that the localization pattern and the depth of the shear zone are shown to depend on system parameters, with a predominance of bottom localization for sufficiently large values of wall friction coefficient and applied pressure. This is important in view of the application of a torsional shear cell as well as an annular shear cell for rheological characterization of granular flows. Recently, we reported [31] on wall forces and on the localization pattern in a linear, periodic geometry which shares some similarities with the configuration studied in the present work and the one studied by Orlando \& Shen [36]. Compared to 
that somewhat ideal configuration, the present study deals with a flow which can be simply realized as an experimental set-up and analysed by means of imaging techniques and force measurements. Future contributions will deal with the analysis of such an experimental setup. In that perspective, the present work will be useful also to determine the range of parameter space to be investigated in order to study experimentally the shear localization in a torsional shear flow.

Acknowledgements The numerical simulations were carried out at the CCIPL (Centre de Calcul Intensif des Pays de la Loire) under the project MTEEGD.

\section{References}

1. Artoni R, Santomaso A (2014) Effective wall slip in chutes and channels: experiments and discrete element simulations. Granular Matter 16(3):377-382

2. Nedderman R, Laohakul C (1980) The thickness of the shear zone of flowing granular materials. Powder Technology 25(1):91 - 100

3. Natarajan VVR, Hunt ML, Taylor ED (1995) Local measurements of velocity fluctuations and diffusion coefficients for a granular material flow. Journal of Fluid Mechanics 304:1-25

4. GDR-MiDi (2004) On dense granular flows. Eur Phys J E 14(4):341-365

5. Singh A, Magnanimo V, Saitoh K, Luding S (2014) Effect of cohesion on shear banding in quasistatic granular materials. Phys Rev E 90:022,202

6. Singh A, Magnanimo V, Saitoh K, Luding S (2015) The role of gravity or pressure and contact stiffness in granular rheology. New Journal of Physics 17(4):043,028

7. Moosavi R, Shaebani MR, Maleki M, Török J, Wolf DE, Losert W (2013) Coexistence and transition between shear zones in slow granular flows. Phys Rev Lett 111:148,301

8. Fenistein D, van Hecke M (2003) Kinematics: Wide shear zones in granular bulk flow. Nature 425(6955):256-256

9. Jop P (2008) Hydrodynamic modeling of granular flows in a modified couette cell. Phys Rev E 77:032,301

10. da Cruz F, Emam S, Prochnow M, Roux JN, Chevoir Fmc (2005) Rheophysics of dense granular materials: Discrete simulation of plane shear flows. Phys Rev E 72:021,309

11. Jop P, Forterre Y, Pouliquen O (2006) A constitutive law for dense granular flows. Nature 441:727730
12. Cortet PP, Bonamy D, Daviaud F, Dauchot O, Dubrulle B, Renouf M (2009) Relevance of viscoplastic theory in a multi-directional inhomogeneous granular flow. EPL (Europhysics Letters) $88(1): 14,001$

13. Brodu N, Richard P, Delannay R (2013) Shallow granular flows down flat frictional channels: Steady flows and longitudinal vortices. Phys Rev E 87:022,202

14. Kamrin K, Koval G (2012) Nonlocal constitutive relation for steady granular flow. Phys Rev Lett 108:178,301

15. Henann DL, Kamrin K (2013) A predictive, sizedependent continuum model for dense granular flows. Proceedings of the National Academy of Sciences 110(17):6730-6735

16. Kamrin K, Henann DL (2015) Nonlocal modeling of granular flows down inclines. Soft Matter 11:179185

17. Henann DL, Kamrin K (2014) Continuum modeling of secondary rheology in dense granular materials. Phys Rev Lett 113:178,001

18. Jenkins J, Berzi D (2010) Dense inclined flows of inelastic spheres: tests of an extension of kinetic theory. Granular Matter 12:151-158

19. Jenkins J, Berzi D (2012) Kinetic theory applied to inclined flows. Granular Matter 14:79-84, 10.1007/s10035-011-0308-x

20. Artoni R, Santomaso A, Canu P (2011) Hysteresis in a hydrodynamic model of dense granular flows. Phys Rev E 83:051,304

21. Jean M (1999) The non-smooth contact dynamics method. Computer Methods in Applied Mechanics and Engineering 177(3-4):235 - 257

22. Renouf M, Dubois F, Alart P (2004) A parallel version of the non smooth contact dynamics algorithm applied to the simulation of granular media. Journal of Computational and Applied Mathematics 168(1-2):375 - 382, selected Papers from the Second International Conference on Advanced Computational Methods in Engineering (ACOMEN 2002)

23. Dippel S, Wolf D (1999) Molecular dynamics simulations of granular chute flow. Computer Physics Communications 121-122:284 - 289, proceedings of the Europhysics Conference on Computational Physics CCP 1998

24. Glasser BJ, Goldhirsch I (2001) Scale dependence, correlations, and fluctuations of stresses in rapid granular flows. Physics of Fluids 13(2):407-420

25. Babic M (1997) Average balance equations for granular materials. International Journal of Engineering Science 35(5):523 - 548 
26. Zhu HP, Yu AB (2002) Averaging method of granular materials. Phys Rev E 66:021,302

27. Weinhart T, Thornton A, Luding S, Bokhove O (2012) From discrete particles to continuum fields near a boundary. Granular Matter 14:289-294

28. Artoni R, Richard P (2015) Average balance equations, scale dependence, and energy cascade for granular materials. Phys Rev E 91:032,202

29. Weinhart T, Hartkamp R, Thornton AR, Luding S (2013) Coarse-grained local and objective continuum description of three-dimensional granular flows down an inclined surface. Physics of Fluids 25(7):070605

30. Luding S (2008) Constitutive relations for the shear band evolution in granular matter under large strain. Particuology 6(6):501 - 505, simulation and Modeling of Particulate Systems

31. Artoni R, Richard P (2015) Effective wall friction in wall-bounded 3d dense granular flows. Phys Rev Lett 115:158,001

32. Koval G, Roux JN, Corfdir A, Chevoir Fmc (2009) Annular shear of cohesionless granular materials: From the inertial to quasistatic regime. Phys Rev E 79:021,306

33. Staron L, Lagrée PY, Popinet S (2014) Continuum simulation of the discharge of the granular silo. The European Physical Journal E 37(1):1-12

34. Byron Bird R, Stewart WE, Lightfoot EN (2007) Transport Phenomena, 2nd edn. Wiley, New-York

35. Unger T, Török J, Kertész J, Wolf DE (2004) Shear band formation in granular media as a variational problem. Phys Rev Lett 92:214,301

36. Orlando A, Shen H (2013) Using the annular shear cell as a rheometer for rapidly sheared granular materials: a dem study. Granular Matter pp 1-12 